\title{
Fast Micromachining Using Spatial Light Modulator and Galvanometer Scanner with Infrared Pulsed Nanosecond Fiber Laser
}

\author{
Jarno J.J. KAAKKUNEN, Ilkka VANTTAJA and Petri LAAKSO \\ VTT Technical Research Centre of Finland, P.O.Box 17021, FI-53851 Lappeenranta, Finland \\ E-mail: jarno.kaakkunen@vtt.fi
}

\begin{abstract}
Rapid method to fabricate microstructures using a combination of a spatial light modulator (SLM) and a galvanometer scanner with an infrared nanosecond fiber lasers is studied. Here the SLM is used as a computer generated hologram which can be applied to modify laser pulses intensity distribution virtually almost arbitrary. SLM is used to separate nanosecond pulses into the several spots so that multiple spots can be machined simultaneously with a fast ablation rate. In this paper it is shown that the micro-machining speeds that can be realized using a combination of the SLM and the scanner, are not always possible to realize using merely scanner. It is shown that about 0.6 million spots per second can be ablated in silicon using this combination.

DOI: $10.2961 /$ jlmn.2014.01.0008
\end{abstract}

Keywords: nanosecond laser, spatial light modulator, computer generated hologram, laser marking, microstructures

\section{Introduction}

Recently, interest of using the liquid crystal on silicon spatial light modulators (Lcos-SLM) in various laser processing applications has increased [1-4]. This is because of the technical development of these adaptive spatial phase elements has enhanced their physical properties, like light utilizing efficiency, amount and size of the pixels. Added to these, technical development had made possible to use them with the lasers that are suitable for the various laser processing applications. Nowadays, they can be applied with both relatively high peak and average power laser pulses $[1,5,6]$.

SLMs can modify the spatially phase of the linearly polarized light and therefore they can be used as an adaptive computer generated holograms (CGH). This enables not only almost arbitrary modification of the laser beams intensity profile but it can be also made online [7]. By using special designing methods it is not only possible to adjust divided spots position in time and 2-dimensional surface but their ablation depth can be also adjusted [8].

Previously, it has been shown that the SLMs can be used with the ultrashort lasers both in visible and IR wavelengths $[1,5]$. Also longer pulses in visible wavelengths have been studied but not in IR wavelengths [6]. In this paper, IR nanosecond pulses are applied with the SLMs using a simple optical configuration. One major benefit of using IR nanosecond laser instead of ultrashort lasers is that their purchase prices are lower. Compared to single pulse ablation using galvanometer scanner (scanner), with the SLM and scanner it is possible to hasten ablation processes, like marking and precise machining, where high fluences are not always tolerated. Usually repetition rate of the laser is the factor that limits the number of marks per second but in case of the SLM main limiting factor is the pulse energy.

\section{Experimental setup and optical design}

Two different lasers were used in studies: IPG YLP-20 and IPG YLP-100. Basic information of these lasers is shown in Table 1. Both of these Gaussian shaped pulse lasers operate with about $1065 \mathrm{~nm}$ wavelength and they are randomly polarized. For both lasers the diameter of the beam is $7.5 \mathrm{~mm}$ after the laser head.

Table 1: Basic information of the lasers used in studies.

\begin{tabular}{|l|c|c|}
\hline & IPG YLP-20 & IPG YLP-100 \\
\hline Pulse length & $110 \mathrm{~ns}$ & $1500 \mathrm{~ns}$ \\
\hline Pulse Energy & $1 \mathrm{~mJ}$ & $2 \mathrm{~mJ}$ \\
\hline Repetition Rate & $20-80 \mathrm{kHz}$ & $5-100 \mathrm{kHz}$ \\
\hline & $20 \mathrm{~W}$ & $100 \mathrm{~W}$ \\
\hline
\end{tabular}

Used liquid crystal on silicon spatial light modulator was X11840-03 from Hamamatsu Photonics. Display of this element consist $792 \times 600$ pixels with a size of the $20 \mu \mathrm{m} \times$ $20 \mu \mathrm{m}$. Fill factor of these pixels is about $95 \%$ and depending on wanted diffraction pattern it can typically utilize about $65 \%-75 \%$ of used laser power. Each pixel can change individually polarization of the incident light with 256 steps and they can cause more than $2 \pi$ phase shift into $1065 \mathrm{~nm}$ wavelength. 


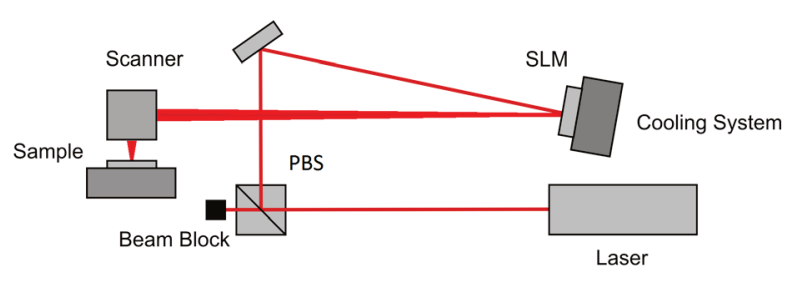

Fig. 1 Schematic of the optical configuration used in micromachining studies.

Schematic of the optical setup used in studies is shown in Figure 1. Polarization of the randomly polarized pulses is first purified using polarizing beam splitter (PBS). Unwanted polarization of the pulses is guided into beam block and therefore half of the lasers power is lost in this point. This means that the maximum available power in SLM with used lasers were $10 \mathrm{~W}$ and $50 \mathrm{~W}$. After PBS pulses are guided with mirror into the SLM with an angle of 3 degree. SLM was cooled from the backside of liquid crystal panel using standard water cooling unit of desktops CPU. From the SLM, pulses are guided into the galvanometer scanner from Scanlab (hurryScan II) with $\mathrm{f}=100 \mathrm{~mm}$ F-theta lens. Distance between SLM and scanner was about $800 \mathrm{~mm}$.

Design of the computer generated holograms were made using self-build Iterative Fourier Transform Algorithm (IFTA) based program made using Matlab [9]. Fresnel lenses were applied in calculations of the CGH $[10,11]$, which makes possible to avoid influence of the $0^{\text {th }}$ diffraction order [12]. With this way the $0^{\text {th }}$-order is focused into different plane than the wanted diffraction pattern. According to manufacturer of the SLM, typical total light utilization efficiency of the SLM in wanted diffraction order is about $70 \%$. This is also taken into account in calculations shown in this paper.

In CGH Fresnel lenses with $\mathrm{f}_{\text {fress }}=-500 \mathrm{~mm}$ were applied. With these parameters the effective focal length of this two-lens system is $\mathrm{f}_{\text {eff }}=41.7 \mathrm{~mm}$. Because the negative Fresnel lens was used, image plane of the wanted diffraction order is about $8.3 \mathrm{~mm}$ after the focus of the $0^{\text {th }}$-order ( $108.3 \mathrm{~mm}$ from the f-theta lens). Because this working area of this double lens system is slightly larger than working area of the f-theta lens. With f-theta lens the working area is about $70 \mathrm{~mm} \times 70 \mathrm{~mm}$, but with the combination of the SLM and f-theta lens the working area is about $75 \mathrm{~mm}$ x $75 \mathrm{~mm}$. However, with used f-theta lens it has been seen that to avoid aberration it is best to keep working area in 50 $\mathrm{mm} \times 50 \mathrm{~mm}$. Therefore the maximum used working area of SLM and scanner system was $55 \mathrm{~mm}$ x $55 \mathrm{~mm}$. Within this working area the no change or distortions on diffraction pattern was observed. Resolution of the used galvanometer scanner was about $10.7 \mu \mathrm{rad}$. This means that without Fresnel lens minimum possible movement is 1.07 $\mu \mathrm{m}$ and with it $1.16 \mu \mathrm{m}$.

\section{Experiments and results}

In first studies, IPG YLP-20 was applied to ablate letters into the steel. In these studies, $20 \mathrm{kHz}$ repetition rate and maximum power ( $\mathrm{P}=10 \mathrm{~W}$ in SLM) were applied. SLM was used to generate $\mathrm{CGH}$ that divides pulses into $\mathrm{V}$ - and T-letters so that each letter consists of 9 spots. These letters

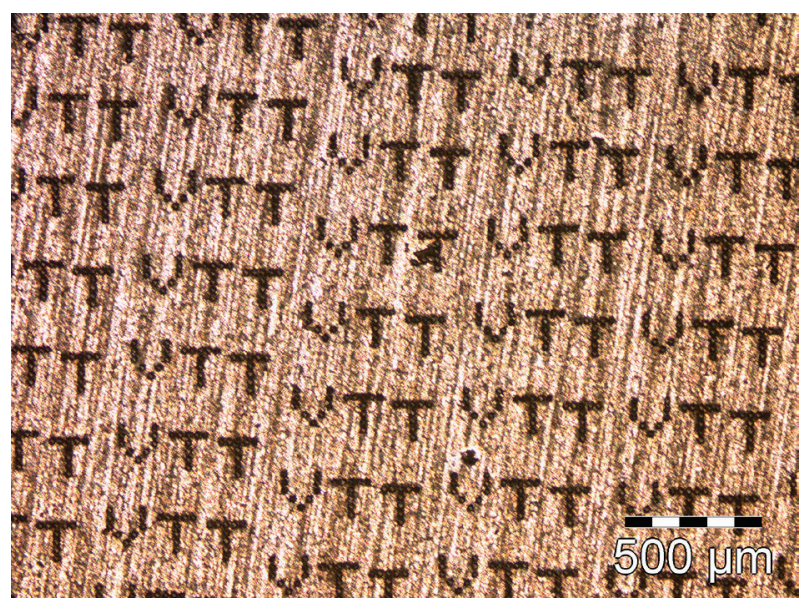

(a)

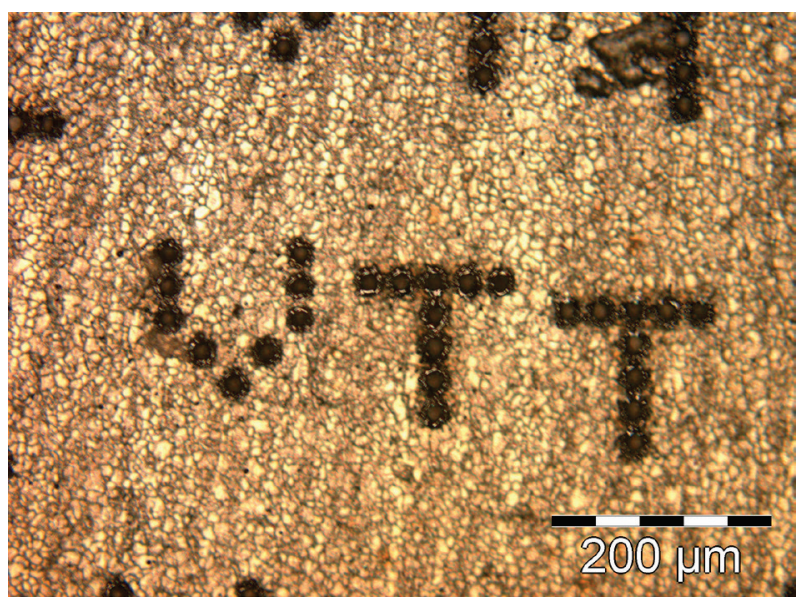

(b)

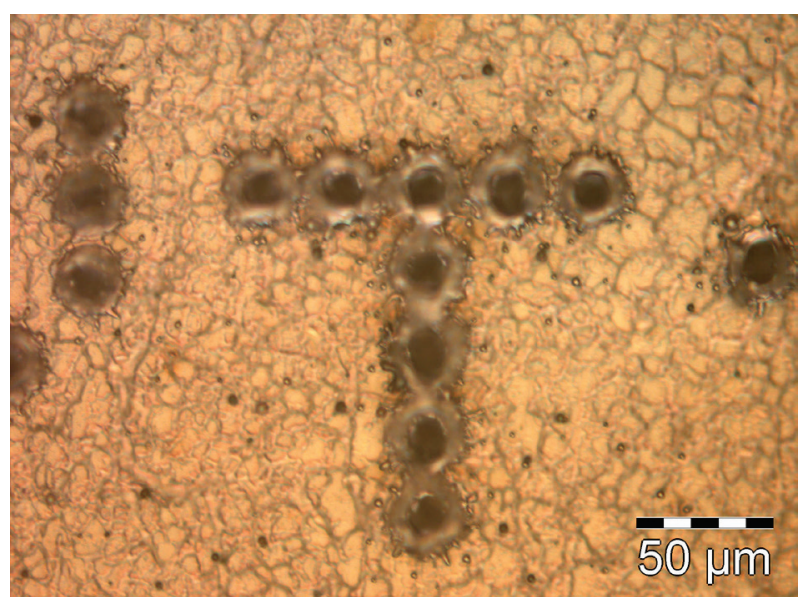

(c)

Fig. 2 Holes ablated using combination of the SLM and galvanometer scanner with IPG YLP-20 in steel. Diameter of the holes is about $25 \mu \mathrm{m}$ and period of them about $30 \mu \mathrm{m}$. Each letter is ablated with single irradiation individually using 4 pulses. Here (b) is magnified image of (a) and (c) is magnification of $(b)$. 
were ablated separately using scanner so that they generate VTT-letter combination. Diameter of the single spot was about $25 \mu \mathrm{m}$ and distances between neighboring spots were about $30 \mu \mathrm{m}$. If we take into account losses in SLM, fluence $F$ can be estimate to be $F=27 \mathrm{~mJ} / \mathrm{cm}^{2}$. Each letter was irradiated separately using four pulses to have visually good contrast in relatively rough surface. Microscope images of these results can be seen in Figure 2. Here (b) is magnification from (a) and (c) from (b). With these parameters about 5000 letters or 45000 holes can be ablated in one second. If similar spacing between letters would be used with this laser it would mean that larger than $25 \mathrm{~mm} \mathrm{x}$ $12 \mathrm{~mm}$ area could be ablated in one second. Naturally same could be realized using merely the scanner but not same ablation speed could be achieved. Although the full repetition rate of the laser $(80 \mathrm{kHz})$ could be used, it would have taken much more time because of the limited maximum speed of scanner.

IPG YLP-20 was also used to ablate periodic hole-arrays into the silicon. Also in this case maximum power $10 \mathrm{~W}$ (in SLM) and repetition rate of $20 \mathrm{kHz}$ was used. With single pulse 15 holes were ablated. Diameter of the holes was about $6 \mu \mathrm{m}$ and period of them about $12 \mu \mathrm{m}$. This means that used fluence was about $F=70 \mathrm{~mJ} / \mathrm{cm}^{2}$. In this case 0.3 million holes per second can be ablated. With these parameters, it means that larger than $6.5 \mathrm{~mm} \times 6.5 \mathrm{~mm}$ area can be ablated in one second.

Ablation of VTT-letter combination was also realized using IPG YLP-100. These results can be seen in Figure 3. In these images, (b) is magnification of (a) and (c) from (b). Here letters are ablated into the silicon using single pulse. Diameter of these single spots was about $7 \mu \mathrm{m}$ and distances between them were about $25 \mu \mathrm{m}$. Here V-letters consists of 11 holes and T-letters 10 holes. In these experiments the maximum power $50 \mathrm{~W}$ (in SLM) and $50 \mathrm{kHz}$ repetition rate was used. This means that in V-letters fluence was $\mathrm{F}=155$ $\mathrm{mJ} / \mathrm{cm}^{2}$ and respectively in T-letters $\mathrm{F}=170 \mathrm{~mJ} / \mathrm{cm}^{2}$. Using these parameters it is possible to ablate 50000 letters or more than 0.5 million holes per second. If similar distances between the letters are used, this means that about $61 \times 19$ $\mathrm{mm}^{2}$ can be ablated in one second. This kind of marking speed is already in limits if merely the standard scanner and laser with faster repetition rate would be used.

Maximum amount of visible dots that could be still ablated with IPG YLP-100 using $50 \mathrm{kHz}$ repetition rate and $50 \mathrm{~W}$ power (in SLM) in silicon was 12. Microscope images of these ablations can be seen in Figure 4. Here (b) is magnified image of (a) and (a) from (c). In this case the fluence was $F=107 \mathrm{~mJ} / \mathrm{cm}^{2}$. Diameter of the holes was under $10 \mu \mathrm{m}$ and period of them about $25 \mu \mathrm{m}$. With these parameters it is possible to ablate 0.6 million holes per second. This means that area of $19 \mathrm{~mm} \times 19 \mathrm{~mm}$ could be ablated in one second if $25 \mu \mathrm{m}$ periods are used. In these experiments hole-array was ablated into the shape of the VTT-logo, see Fig. 4 (c). Making this took only few seconds. As it can be seen, the VTT-logo visually diffracts light because the small enough periodic structure.

\section{Discussion and Conclusions}

Usage of the infrared pulsed nanosecond fiber laser with the spatial light modulator using the galvanometer scanner was shown. SLM was used as an adaptive comput-

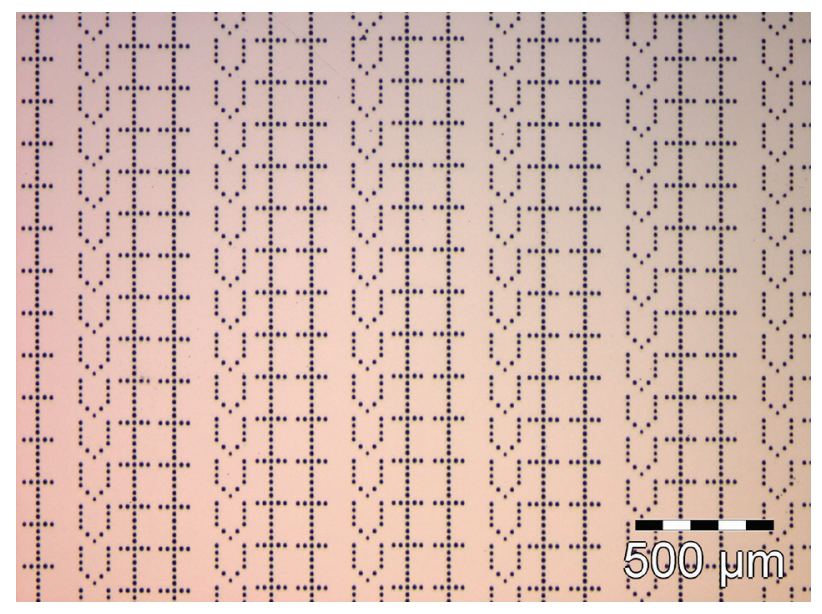

(a)

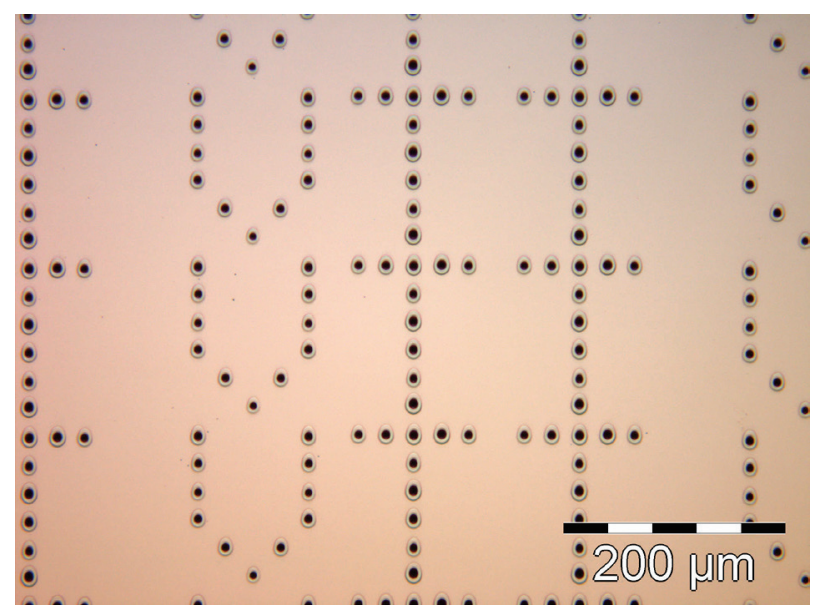

(b)

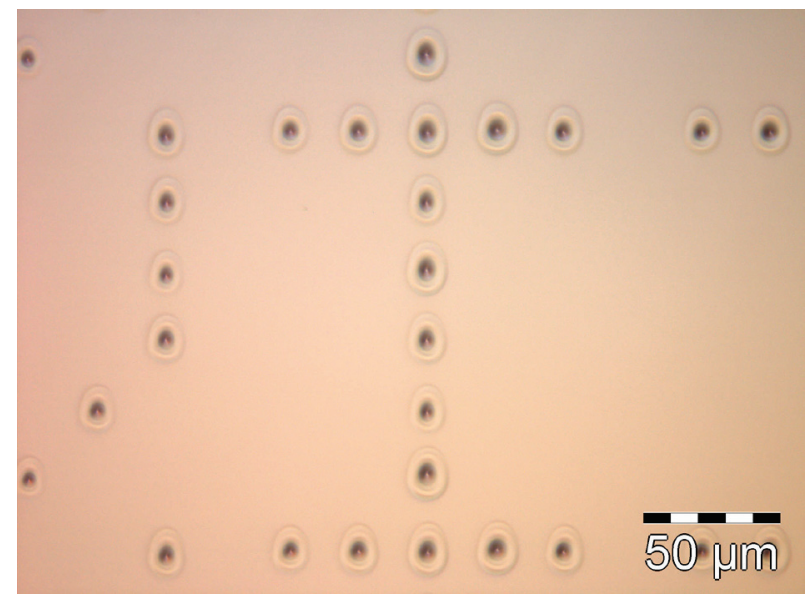

(c)

Fig. 3 Optical microscope images of VTT-letter combination ablated into silicon. Each letter is ablated separately using single pulse. Diameter of the holes is about $7 \mu \mathrm{m}$ and distance between contiguous dots is $25 \mu \mathrm{m}$. (b) is magnification of (a) and (c) from (b). 


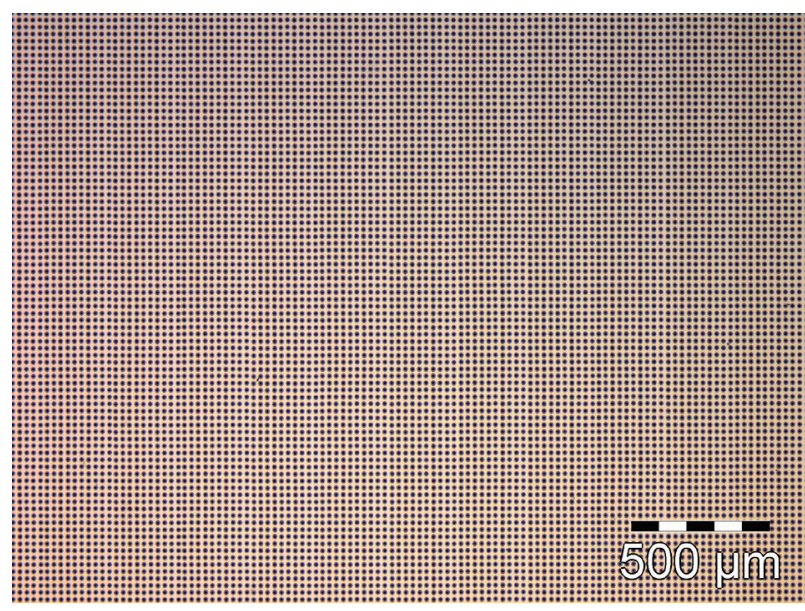

(a)

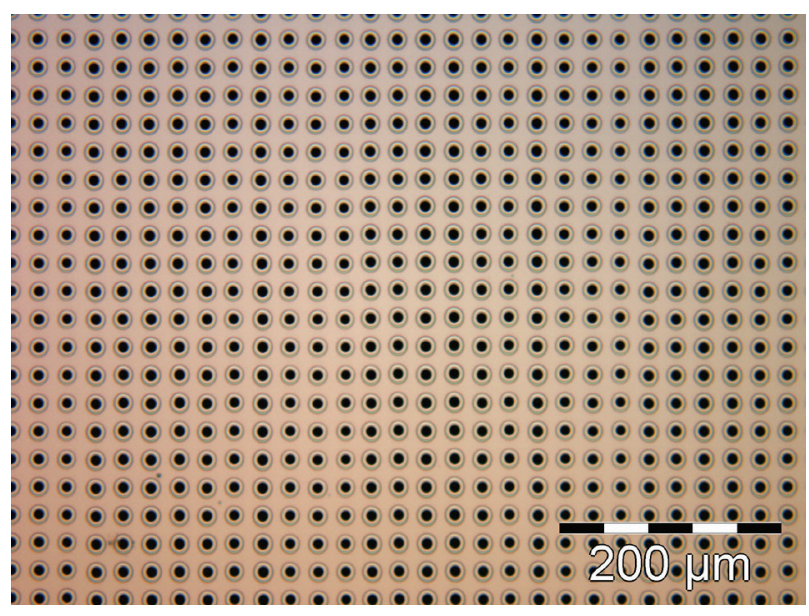

(b)

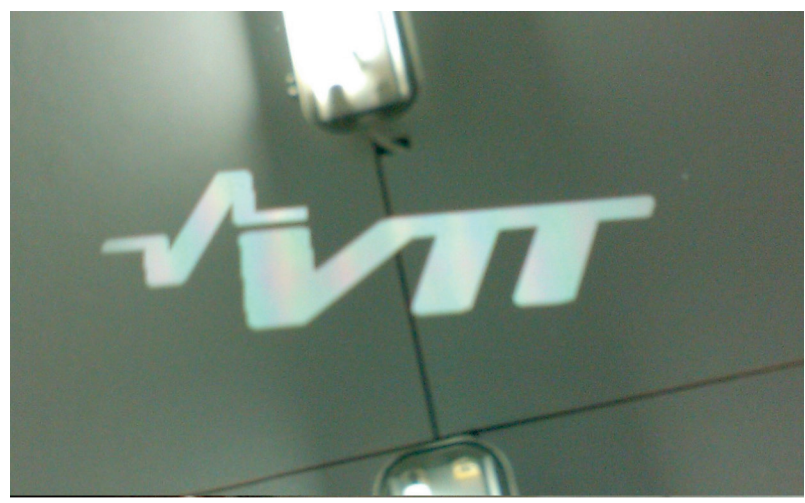

(c)

Fig. 4 Optical microscope images of dot-matrix array ablated into silicon. Diameter of the holes is under $10 \mu \mathrm{m}$ and period of them is $25 \mu \mathrm{m}$. Here (a) is magnification of (c) and (b) from (a). Dot matrix array was ablated into shape of the VTT-logo (c). In this logo, visual diffraction effect can be observed because of the small enough periodic structure.

er generated hologram. CGH was used to divide pulses into several spots so that multiple marks could be ablated simultaneously. It was shown that with this kind of combination it is possible to achieve such ablation rates that are not al- ways possible to handle when using merely scanner. In studies it was shown that about 0.6 million holes per second could be done in one second. This kind of ablation speed is already in limits or even over if merely faster laser and standard galvanometer scanner would be used. It might be still possible to ablate holes near to each other but holes relatively far from each other would be impossible.

In these experiments only shallow structures were studied. Naturally if more pulses would be applied SLM would be still faster option than merely galvanometer scanner. In case of the IPG YLP-100 in these experiments it was seen that even if we do not take into account scanner movements SLM is about 6 times faster than simple scanner. Naturally if we would take into account the scanner movements this factor would be larger.

In our studies, lasers with randomly polarized pulses were used. This causes that only half of the laser power can be utilized into the wanted diffraction pattern, because SLM can only modify phase of the linear polarized light. Therefore lasers used in studies are not optimal to be used with SLMs but unfortunately no better lasers were available for the studies.

During the studies it was seen that cooled SLM can handle more than $50 \mathrm{~W}$ average power of nanosecond pulsed laser irradiation so even more could be applied with it. Therefore in a future, nanosecond lasers with more power will be tested with this kind of SLM. We believe that ablation rate of the hole arrays can be hastened with more than factor of 10. Added to laser marking and precise micro-machining this also opens new possibilities for usage SLM in a new field of laser applications.

\section{Acknowledgments and Appendixes}

This study was financed by national research project MULTIBEAM. The authors would like to thank Finnish Funding Agency for Technology and Innovation (TEKES) for their founding. Cencorp Corporation is appreciated for loaning the IPG YLP-100.

\section{References}

[1] Z. Kuang, W. Perrie, J. Leach, M. Sharp, S.P. Edwardson, M. Padgett, G. Dearden and K.G. Watkins, Applied Surface Science, 255 (2008), 2284-2289.

[2] H. Takahashi, S. Hasegawa, A. Takita and Y. Hayasaki, Optics Express, 16 (2008), 16592-16599.

[3] Z. Kuang, W. Perrie, D. Liu, S. Edwardson, J. Cheng, G. Dearden and K. Watkins, Applied Surface Science 255 (2009), 9040-9044.

[4] M. Sakakura, T. Sawano, Y. Shimotsuma, K. Miura and K. Hirao, Optics Express, 18 (2010), 12136-12143

[5] Y. Hayasaki, T. Sugimoto, A. Takita and N. Nishida, Applied Physics Letters, 87 (2005), 031101.

[6] R.J. Beck, J.P. Parry, W.N. MacPherson, A. Waddie, N.J. Weston, J.D. Shephard and D. Hand, Optics Express, 18 (2010), 17059-17065.

[7] J. Turunen and F. Wyrowski: "Diffractive Optics: Industrail and Commercial Applications", (Akademic Verlag 1998), p.440.

[8] M. Yamaji, H. Kawashima, J. Suzuki and S. Tanaka, Applied Physics Latters, 93 (2008), 041116.

[9] F. Wyrowski and O. Bryngdahl, Journal of Optical Society of America A, 5 (1988), 1058-1065. 
[10] S. Hasegawa and Y. Hayasaki, Optical Review, 14 (2007), 208-2013.

[11] S. Hasegawa, Y. Hayasaki, T. Sugimoto, and N. Nishida, Optics Letters, 31 (2006), 1705-1707.
[12]Z. Kuang, D. Liu, W. Perrie, S. Edwardson, M. Sharp,

E. Fearon, G. Dearden and K. Watkins, Applied Surface Science 255 (2009), 6582-6588.

(Received: June 28, 2013, Accepted: December 30, 2013) 